\title{
Pleural effusion: Presentation, causes and treatment outcome in a resource limited area, Ethiopia
}

\author{
Mekonnen Desalew ${ }^{1}$, Amare Amanuel $^{2}$, Alemu Addis $^{3}$, Hurissa Zewdu $^{1}$, Ali Jemal $^{4}$ \\ ${ }^{1}$ Department of Internal Medicine, University of Gondar Ethiopia, Gondar, Ethiopia; ${ }^{*}$ Corresponding Author: desalewm@yahoo.com \\ ${ }^{2}$ Department of Neurology, Addis Ababa University, Addis Ababa, Ethiopia, Ethiopia \\ ${ }^{3}$ CU-ICAP, Addis Ababa, Ethiopia \\ ${ }^{4}$ Department of Microbiology, Immunology and Parasitology, University of Gondar, Gondar, Ethiopia
}

Received 23 October 2011; revised 15 November 2011; accepted 12 December 2011

\section{ABSTRACT}

Background: Pleural effusion is a common clinical problem with different causes. Objective: To demonstrate clinical features and outcome of pleural effusion. Methods: Prospective descriptive study was conducted involving $\mathbf{1 1 0}$ patients with pleural effusion admitted to a resource limited hospital in Ethiopia. Results: Males and females were almost equally represented. Cough, fever and weight loss were prominent presenting symptoms accounting 90, 77.3 and 77.3 percent respectively. Right side effusion was the common presentation 50 (45.5\%). Forty (37.4\%) patients had HIV infection among 107 tested. Tuberculosis was the commonest cause 78 (70.9\%) followed by parapneumonic effusion 36 (32.7\%) and empyema 27 (24.5\%). Malignant pleural effusion was detected only in one patient. Eighty one (73.6\%) improved from their illness and 7 (6.4\%) died. Lympocytic pleural effusion found to be associated with tuberculosis (OR $=\mathbf{3 . 9 4 2}$ (1.527 - 10.179), $P=0.005$. There were no associations between HIV infection, anemia, elevated ESR and side of pleural effusion with tuberculosis. Conclusion: Tuberculosis was the leading cause of pleural effusion in our setup even though etiologic diagnosis was difficult. Strengthening the laboratory and pathology services in the area is strongly recommended.

Keywords: Pleural Effusion; Tuberculosis;

Parapneumonic Effusion; Empyema; Pleura

\section{INTRODUCTION}

Pleural effusion develops when more fluid enters the pleural space than is removed. It is a common clinical problem with different possible causes. It can be due to local or systemic or infectious or non-infectious causes.
Patients with pleural effusion have higher morbidity and mortality than patients without pleural effusion [1].

A definitive diagnosis, as is provided by the finding of malignant cells or specific organism in the pleural fluid can be established in approximately $25 \%$ of cases. However, a presumptive diagnosis, based on the prethoracentesis clinical impression and pleural effusion analysis, clinical decision-making information can be gained in over $90 \%$ of patients [2]. Diagnosis of the cause is often difficult. The relative frequency of causes of pleural effusion is known to vary in different parts of the world [3]. However, in developing nations infections-especially tuberculosis and parapneumonic effusions are more prevalent [4].

Pleural effusions are frequently encountered in hospitalized HIV/AIDS patients [5]. Among patients with tuberculosis pleural involvement appears to be more common when co-infection with HIV is present [6].

There were no similar studies published in the area and this study was conducted to fill this gap. This prospective descriptive study demonstrates the presentation, causes and treatment outcome of patients presented to Gondar University Hospital with pleural effusion.

\section{PATIENTS AND METHODS}

The prospective study was conducted at Gondar University Hospital. It is a tertiary teaching hospital with 400 beds. The study include all patients with pleural effusion admitted to the medical wards of Gondar University Hospital from Jan 2006 to Nov 2007 who gave an informed written consent and fulfill the inclusion criteria.

\subsection{Inclusion Criteria}

Adult patients (age $\geq 13$ years) presented to Gondar University Hospital with pleural effusion who required diagnostic thoracentesis were included to the study.

\subsection{Ethical Clearance}

Research protocol was approved by the Research and 
Publication Office of the University of Gondar. Permission to proceed the study was obtained from the medical director of Gondar University Hospital. Confidentiality was maintained while using the patients' data. Informed written consent was obtained from each patient and the information was analyzed anonymously.

\subsection{Data Collection}

The following data were collected using data sheet for each patient who qualifies for pleural effusion: sociodemographic, clinical findings, underlying disease, duration of symptoms, duration of hospital stay, Chest $\mathrm{X}$ ray, hematocrit, erythrocyte sedimentation rate, pleural fluid analysis (cell count differential count, cytology and microbiologic analysis) and HIV serology. We also collected data on treatments given including antibiotics, anti tuberculosis and tube thoracostomy. We also followed patients during their stay at the hospital and outcome variables and complications were noted. A pretested structured questionnaire was used to collect data. The questionnaire was prepared by the investigators for this study purpose. It was pretested and several revisions were made before its application.

Outcome variables compiled as "improved" were patients who got clinical improvement as a response to treatment and "same" were patients who were discharged against medical advice, disappeared from the hospital and those referred to other center for further evaluation and management. Those with "deterioration" were worsening clinical condition despite treatment. "Deaths" were ascribed to hospital deaths while patients were on treatment.

\subsection{Statistical Analysis}

SPSS 12.1 computer software was used for data entry and analysis. $\mathrm{P}$ value less than 0.05 was used for statistical significance. Associations were assessed using binary and multinomial logistic regression.

\section{RESULTS}

In this prospective descriptive study, we assessed 110 patients with pleural effusion admitted to Gondar University Hospital from Jan 2006 to Nov 2007.

The sociodemographic characteristics are described in Table 1. The number of male and female patients in the study was comparable (54 and 56 respectively). The mean age was 37 years with the range of $63(15-78)$. Seventy two $(65.5 \%)$ patients were age under 40 years.

The mean duration of the clinical symptoms prior to presentation was 66 days with a range of 362 days ( 3 to $365)$. The mean hospital stay was 25 days with a range of 100 days (3 to 103 ).
Table 1. Age and Sex distribution of 110 patients with pleural effusion admitted to Gondar University Hospital from Jan. 2006 to Nov. 2007.

\begin{tabular}{|c|c|c|c|c|}
\hline \multirow[b]{2}{*}{ Age Group } & \multicolumn{2}{|c|}{ Sex } & \multirow{2}{*}{$\begin{array}{l}\text { Total Number } \\
\quad(\mathrm{n}=110)\end{array}$} & \multirow[b]{2}{*}{ Total Percent } \\
\hline & $\begin{array}{c}\text { Male } \\
(\mathrm{n}=54)\end{array}$ & $\begin{array}{l}\text { Female } \\
(\mathrm{n}=56)\end{array}$ & & \\
\hline $15-20$ & 11 & 7 & 18 & 16.4 \\
\hline $21-30$ & 13 & 23 & 36 & 32.7 \\
\hline $31-40$ & 10 & 8 & 18 & 16.4 \\
\hline $41-50$ & 6 & 11 & 17 & 15.5 \\
\hline $51-60$ & 6 & 3 & 9 & 8.2 \\
\hline $61-70$ & 5 & 3 & 8 & 7.3 \\
\hline $71-78$ & 3 & 1 & 4 & 3.6 \\
\hline
\end{tabular}
2.

The presenting symptoms were represented with Table

Twenty one (19.1\%) patients had history of previous treatment for tuberculosis. Previous history of pleural effusion was reported in $4(3.6 \%)$ patients.

On physical examination, body swelling was traced in $43(39.1 \%)$ of patients which comprised 39 patients had leg edema, 22 patients had ascites and 4 patients had peri orbital edema. Clinically significant lymphadenopathy was found in $8(7.3 \%)$ of patients.

Comorbidity with underlying chronic illness was found in $23(20.9 \%)$ patients comprising 3 hypertensive, 8 congestive heart failure, 2 chronic liver disease, 2 renal failure and 6 with other chronic illnesses. In this description, one patient might have more than one comorbid chronic illness.

Right sided accounting 50 (45.5\%) followed by left sided $41(37.3 \%)$ then bilateral $19(17.3 \%)$.

Underlying HIV infection was found in 40 (37.4\%) patients out of 107 tested. Twelve patients were taking antiretroviral therapy.

Chest X-ray findings of 110 patients reported were described in Table 3.

Appearance of pleural fluid was documented in 100 patients and majority of them had straw colored 70 $(66 \%)$, pus $24(22.6 \%)$ and $6(5.7 \%)$ had hemorrhagic effusion.

On the pleural fluid analysis, lymphocytes were predominant in $57(64.8 \%)$ and neutrophils predominate in $31(35.2 \%)$ samples.

There were only three pleural fluid samples among 88 stained with gram stain showing one gram negative rods, one gram positive cocci and one mixed bacteria of gram positive cocci and gram negative rods. Culture of the pleural fluid among 88 samples grew bacteria in only three of the cases which were S. aureus in two cases and S. epidermidis in one case.

Pleural fluid cytology was done for 27 patients and lymphocytic effusion reported in 16 patients followed by 
Table 2. Presenting symptoms of 110 patients with pleural effusion admitted to Gondar University Hospital from Jan. 2006 to Nov. 2007.

\begin{tabular}{lcc}
\hline Symptoms & Number & Percent \\
\hline Cough & 99 & 90 \\
Fever & 85 & 77.3 \\
Weight loss & 85 & 77.3 \\
Night sweating & 80 & 72.7 \\
Chest pain & 77 & 70 \\
Sputum production & 73 & 66.4 \\
Dyspnoea & 64 & 58.2 \\
Hemoptysis & 16 & 14.5 \\
\hline
\end{tabular}

Table 3. Chest X-ray findings in 110 patients with pleural effusion admitted to Gondar University Hospital from Jan. 2006 to Nov. $2007^{\S}$.

\begin{tabular}{lcc}
\hline Chest X-ray findings & Number & Percent \\
\hline Right pleural effusion & 50 & 45.5 \\
Left pleural effusion & 41 & 37.3 \\
Bilateral pleural effusion & 19 & 17.3 \\
Hydropneumothorax & 4 & 3.6 \\
Loculated effusion & 3 & 2.7 \\
Consolidation & 4 & 3.6 \\
Parenchymal infiltrates & 3 & 2.7 \\
\hline
\end{tabular}

${ }^{\S}$ One chest $\mathrm{x}$-ray may have more than one feature.

empyema in 5 patients. The others were 4 patients with reactive effusion and 2 were non conclusive.

Hematocrit level was determined for 97 patients and 64 (66\%) had anemia. Erythrocyte Sedimentation Rate (ESR) was elevated in 52 patients $(80 \%)$ among the 65 patients determined.

Table 4 represents final diagnosis of studied patients with pleural effusion.

Of the 78 patients with tuberculosis, 38 had right sided pleural effusion followed by 29 with left sided and 11 patients had bilateral pleural effusion.

Among the 40 HIV/AIDS patients reported 30 had tuberculosis, 8 had empyema and 2 had para pneumonic effusion.

Anti-tuberculosis and antibiotics were given to 79 (71.8\%) and $72(65.5 \%)$ patients respectively. Chest tube drainage was done in $25(22.7 \%)$ patients. Two patients with empyema died before chest tube was inserted.

Out come at hospital discharge was improved in 81 (73.6\%), same in $17(15.5 \%)$, deteriorated with out death in $5(4.5 \%)$ and $7(6.4 \%)$ died while they were in the hospital.

Of the 7 deaths encountered 4 had tuberculosis, 2 had empyema and 1 had constrictive pericarditis. Three of the deceased had HIV infection. Causes of death were
Table 4. Final diagnosis of 110 patients with pleural effusion in patients admitted to Gondar University Hospital from Jan. 2006 to Nov. $2007^{*}$.

\begin{tabular}{lcc}
\hline Diagnosis & Number & Percent \\
\hline Tuberculosis $^{ \pm}$ & 78 & 70.9 \\
Parapneumonic effusion $^{\$ \pi}$ & 36 & 32.7 \\
Empyema $^{\text {Visceral Leishmaniasis }}$ & 27 & 24.5 \\
Heart failure & 10 & 9.1 \\
Lymphoma & 8 & 7.3 \\
Chronic liver disease & 4 & 3.6 \\
Renal failure & 2 & 1.8 \\
Metastasis & 2 & 1.8 \\
Constrictive pericarditis & 1 & 0.9 \\
\hline
\end{tabular}

* One patient may have more than one diagnosis; ${ }^{ \pm}$The diagnosis of TB was based upon clinical assessment, chest X-ray findings, suggestive pleural fluid analysis and responses to anti-TB chemotherapy; ${ }^{\S}$ Empyema was diagnosed based on the finding of gross pus appearance or pleural fluid culture or Gram stain showing organisms; "Among 27 patients with empyema 14 were due to tuberculosis and 13 were due to complicated pneumonia.

respiratory failure related to underlying pleural effusion in 4 cases, one with sepsis related multi organ failure, one with complicated fluid electrolyte imbalance related to abdominal surgery and one patient with constrictive pericarditis died of heart failure precipitated by hospital acquired pneumonia. Two of the cases complicated by respiratory failure were having empyema and they died before chest tube was inserted.

\section{DISCUSSION}

In our study the single most important cause of pleural effusion was tuberculosis accounting 78 (70.9\%) of patients as compared to other Ethiopian studies assessing causes of empyema demonstrated as 59.1\% at Tikur Anbessa Specialized teaching hospital in Addis Ababa [7] and 56\% at Gondar University Hospital [8]. Among empyema cases in our study 14/27 (51.9\%) had tuberculosis which is comparable to the above empyema studies in Ethiopia. The second cause of pleural effusion is complicated pneumonia with parapneumic effusion accounting $32.7 \%$ as comparable to a study conducted in Ghana (21.2\%) [4]. Infectious causes of pleural effusion are still common causes of pleural effusion in developing countries as described also in other studies [4,9], unlike data from developed countries where primary and secondary malignancies outnumbered [10].

The majority of patients were ages below 40 years old $(65.5 \%)$ which is consistent with the above mentioned Ethiopian studies $81.6 \%$ in Addis Ababa [7] and $82 \%$ of patients below the age of 50 years at Gondar [8] and also can be explained by the general population of Ethiopia 
[11].

The commonest symptoms of patients with pleural effusion were cough $(90 \%)$, fever $(77.3 \%)$, weight loss $(77.3 \%)$ and chest pain $(70 \%)$ which is in agreement with other studies $(4,9,10)$.

The magnitude of HIV infection in pleural effusion due to tuberculosis patients were $38.5 \%(30 / 78)$ as compared to $55.5 \%$ in a study conducted at Ghana [4] and $61.4 \%$ in a study at New York [12]. Among $40 \mathrm{HIV}$ positive patients with pleural effusion 30 had tuberculosis. We didn't get statistically significant associations between HIV infection and tuberculosis as a cause of pleural effusion in our study.

Lympocytic pleural effusion accounted in $64.8 \%$ patients and was statistically associated with tuberculosis (Table 5).

We didn't get statistically significant associations between tuberculosis and side of effusion, anemia, elevated ESR and HIV infection with individual binary and multinomial logistic regression (Table 5).

There was a diagnostic constraint observed in our study especially on pleural fluid protein, glucose, lactate dehydrogenase (LDH) which has vital role in classifying the fluid as exudative and transudative. Fluid cytology reports were not also complete due to lack of sustained pathology service. Pleural biopsy was not also done in any patient. Advanced tests like adenosine diaminase (ADA) and interferon (IFN) were not available in the country as a whole. Due to this incomplete laboratory data, classification of pleural fluid to transudative and exudative was unattempted in this study [8].

Pleural fluid microbiologic studies were not satisfactorily providing evidences. There were only three specimens gram stain demonstrate bacteria and three specimens grew bacteria on culture. Acid fast stain didn't demonstrate organism among 88 samples examined.

Table 5. The characteristics of tuberculous and non tuberculous effusions in patients admitted to Gondar University Hospital from Jan. 2006 to Nov. 2007.

\begin{tabular}{|c|c|c|}
\hline \multirow{2}{*}{ Characteristics } & Tuberculosis & Non tuberculosis \\
\hline & $(\mathrm{n}=78)$ & $(\mathrm{n}=32)$ \\
\hline Right sided effusion & $38(48.7 \%)$ & $12(37.5 \%)$ \\
\hline Left sided effusion & $29(37.2 \%)$ & $12(37.5 \%)$ \\
\hline Bilateral effusion & $11(14 \%)$ & $8(25 \%)$ \\
\hline HIV positive ${ }^{*}$ & 30 & 10 \\
\hline Anemia $^{\S^{*}}$ & 48 & 16 \\
\hline Elevated ESR ${ }^{*}$ & 40 & 12 \\
\hline Lymphocytic effusion $^{{ }^{*}}$ & 48 & 9 \\
\hline
\end{tabular}

* Complete data for HIV status, hematocrit level, ESR level and pleural fluid analysis for lymphocytes were not available to demonstrate comparisons percentages; ${ }^{8}$ Hematocrit level below 36 for females and 39 for males were taken as anemia [13]; ${ }^{\sharp}$ Lymphocytic effusion was defined as lymphocyte percentage greater than $50 \%$ [14].
In conclusion, tuberculosis is the leading cause of pleural effusion in our study and it is wise to consider it in a resource limited setups. Empirical therapy with anti tuberculosis chemotherapy in patients with undiagnosed pleural effusion should be taken as a last resort [15]. Laboratory and pathology services have vital contribution for etiologic diagnosis of pleural effusion and it is recommended to strengthen the service in the area [8].

\section{ACKNOWLEDGEMENTS}

We acknowledge the Research and Publications Office of the University of Gondar for funding this study. Many thanks go to all patients who participated in the study. We are also equally grateful to all staff members of the department of internal medicine of Gondar University Hospital for their multidisciplinary support.

\section{REFERENCES}

[1] Khan, J. and Ellis, M.E. (1997) Anaerobic bacterial pneumonia, lung abscess, pleural effusion/empyema. In: Ellis M. E. Ed., Infectious Disease of the Respiratory Tract. Cambridge University Press, Cambridge, 358-373.

[2] Collins, T.R. and Sahn, S.A. (1987) Thoracocentesis: Clinical value, complications, technical problems, and patient experience. Chest, 91, 817-822. doi:10.1378/chest.91.6.817

[3] Storey, D.D., Dines, D.E. and Coles, D.T. (1976) Pleural effusion, a diagnostic dilemma. JAMA, 236, 2183-2186. doi:10.1001/jama.1976.03270200021022

[4] Afful, B., Murphy, S., Antunes, G. and Dudzevicius, V. (2008) The characteristics and causes of pleural effusions in Kumasi Ghana, a prospective study. Tropical Doctor, 38, 219-220. doi:10.1258/td.2007.070275

[5] Joseph, J., Strange, C. and Sahn, S.A. (1993) Pleural effusion in hospitalized patients with AIDS. Annals of Internal Medicine, 118, 856-859.

[6] Frye, M.D., Pozsik, C.J., Sahn S.A. (1997) Tuberculous pleurisy is more common in AIDS than non-AIDS patients with tuberculosis. Chest, 112, 393-397. doi:10.1378/chest.112.2.393

[7] Ali, A., Biluts, H. and Gulilat, D. (2003) Managment of empyema thoracis in Tikur Anbessa Hospital. A three year experience. East and Central Journal of Surgery, 1, 47-50.

[8] Amare, A., Ayele, B. and Mekonnen, D. (2010) Empyema thoracis: Presentation and treatment outcome at gondar university hospital, Northwest Ethiopia. East and Central Journal of Surgery, 1, 119-123.

[9] Al-Alusi F.A. (1986) Pleural effusion in Iraq: A prospective study of 100 cases. Thorax, $\mathbf{4 1}, 492-493$.

[10] Porcel, J.M. and Vives, M. (2003) Ethiology and pleural fluid characterstics of large and massive effusions. Chest, 124, 978-983.

[11] Central Statstical Agency. (2005) Ethiopian demographic and health survey. Population pyramid, Addis Ababa, p. 
14. doi: $10.1136 /$ thx. 41.6 .492

[12] Relkin, F. and Aranda, C.P. (1994) Pleural tuberculosis and HIV infection. Chest, 105, 1338-1341. doi: $10.1378 /$ chest.124.3.978

[13] World Health Organization. (1968) Nutritional anemias: Report of a WHO scientific group. Switzerland. World Health Organization, Geneva.
[14] Porcel, J.M. and Light, R.W. (2006) Diagnostic approach to pleural effusion in adults. American Family Physician, 73, 1211-1220. doi:10.1378/chest.105.5.1338

[15] Sane, M. et al. (2007) Empiric antituberculous therapy in patients with unexplained exsudative pleural effusion. Is it valid in Senegal? Revue De Pneumologie Clinique, 63, 247-250. doi:10.1016/S0761-8417(07)92647-4 